\title{
The safety and effectiveness of chenodeoxycholic acid treatment in patients with cerebrotendinous xanthomatosis: two retrospective cohort studies
}

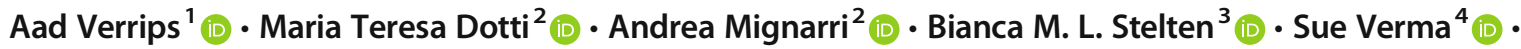 \\ Antonio Federico ${ }^{2}$ (D)
}

Received: 4 October 2019 / Accepted: 20 November 2019 / Published online: 20 December 2019

(C) The Author(s) 2019

\begin{abstract}
Objective To evaluate the safety and effectiveness of chenodeoxycholic acid (CDCA) treatment in patients with cerebrotendinous xanthomatosis (CTX).

Methods Two retrospective cohort studies were conducted in CTX patients who underwent CDCA treatment: one in the Netherlands (NL; CDCA-STUK-15-001) and one in Italy (IT; CDCA-STRCH-CR-14-001). Eligible patients were aged 2-75 years, had been diagnosed with CTX, and were treated with CDCA orally for $\geq 1$ year. The impact of CDCA treatment on biochemical markers (including serum cholestanol levels) and disease signs and symptoms were assessed, in addition to the safety and tolerability of CDCA treatment.

Results A total of 35 patients were screened in the NL study and were diagnosed with CTX at $25.6( \pm 13.7 \mathrm{SD})$ years on average. These patients were treated with CDCA and followed up for a median of 9.00 (range: 0.4-26.3) years. In addition, 28 patients were enrolled in the IT study and were diagnosed at 35.0 ( $\pm 11.4 \mathrm{SD}$ ) years on average (median duration of CDCA treatment: 5.75 [range: 0.0-25.0] years). Signs and symptoms of disease resolved, improved, or remained stable in many patients, with concomitant improvements in biochemical marker levels (serum cholestanol, $p<0.001 ; 7 \alpha$-hydroxy-4-cholesten-3-one, $p<0.001$ [IT study]).

Conclusions The outcomes of these retrospective cohort studies indicate that CDCA is effective in the long-term treatment of CTX, with an acceptable safety profile.
\end{abstract}

Keywords Chenodeoxycholic acid $\cdot$ Cerebrotendinous xanthomatosis $\cdot$ Metabolic disorders $\cdot$ Italy $\cdot$ The Netherlands

\section{Introduction}

Cerebrotendinous xanthomatosis (CTX; OMIM 213700) is a rare, autosomal recessive disorder caused by mutations in

Electronic supplementary material The online version of this article (https://doi.org/10.1007/s10072-019-04169-8) contains supplementary material, which is available to authorized users.

\section{Aad Verrips}

A.Verrips@cwz.nl

Canisius-Wilhelmina Hospital, Nijmegen, the Netherlands

2 Department of Medicine, Surgery and Neuroscience, University of Siena, Siena, Italy

3 Catharina Hospital, Eindhoven, the Netherlands

4 Leadiant Biosciences Ltd., London, UK
CYP27A1, which lead to a deficiency in sterol 27 hydroxylase $[1,2]$. The latter is important for primary bile acid synthesis, specifically the conversion of cholesterol to chenodeoxycholic acid (CDCA) and cholic acid [2, 3]. A deficiency in sterol 27-hydroxylase leads to reduced levels of CDCA; however, patients may have unaffected or higher levels of cholic acid $[2,4]$. In addition, CTX patients have high levels of plasma cholestanol, which accumulates in bile and various tissues, particularly the brain [2].

Manifestations of CTX can vary, not only within families but even between twins [5]. These include prolonged neonatal jaundice, infantile-onset chronic diarrhoea, juvenile cataracts, tendon xanthomas with onset during adolescence or young adulthood, osteoporosis, and progressive psychiatric and neurological impairment $[2,6]$. The latter may include mental retardation, cerebellar syndrome, pyramidal syndrome, peripheral neuropathy, epileptic seizures and dementia [2]. The 
accumulation of cholestanol is thought to be responsible for these neurodegenerative symptoms [2]. Progressive neurological and psychiatric dysfunction are the most disabling features of CTX [4].

More than 300 patients have been diagnosed with CTX worldwide, although the condition is likely to be underdiagnosed [2]. The estimated prevalence of CTX in white populations is 1:50,000; however, this is limited to patients with the R362C CYP27A1 mutation [7]. The diagnosis of CTX typically involves biochemical testing for plasma cholestanol levels, which in CTX are usually markedly increased [2]. The normal range for cholestanol is $3.37 \pm 1.55 \mathrm{mg} / \mathrm{L}$ $(8.66 \pm 3.98 \mu \mathrm{mol} / \mathrm{L})$, but in CTX patients it may be several times higher than this [8-10]. Elevated plasma concentration of the bile acid precursor $7 \alpha$-hydroxy-4-cholesten-3one has also been suggested as a new diagnostic marker of CTX, although these tests are not widely available $[8,11]$. Urinary bile alcohol levels can also be used for diagnosis, although genetic testing for mutations in the CYP27A1 gene is the gold standard and is generally used for confirmation of diagnosis [2, 8].

CDCA has become the standard of care for CTX patients [4]. It prevents the accumulation of cholestanol by inhibiting bile acid synthesis through a negative feedback pathway [12]. Exogenous CDCA has been shown to improve both biochemical and clinical outcomes in CTX patients [4]. Given the natural course of CTX, the primary aim of treatment is stabilisation or improvement of neurological signs and symptoms [4]. Nevertheless, a delay in diagnosis and treatment can lead to a worse prognosis, irreversible neurological damage and severe disability [13].

A recent study by Stelten et al. (2018) demonstrated that starting treatment at an early age can reverse and prevent neurological symptoms [14]. The authors reported results from a retrospective study performed in the Netherlands that evaluated the effect of CDCA on disease progression in 56 CTX patients. Disease progression was measured through correlation between age at diagnosis and clinical characteristics, and with the course of modified Rankin Scale and Expanded Disability Status Scale (EDSS) scores at follow-up [14].

Here, we present data from two retrospective studies, submitted for regulatory purposes to the EMA, in CTX patients who underwent CDCA treatment. The aims of both studies were to evaluate the impact of CDCA on serum cholestanol levels (among other biochemical markers), the effectiveness of this treatment for ameliorating the signs and symptoms of disease, and the impact on disability scores over time. We also retrospectively evaluated the safety and tolerability of CDCA treatment in CTX patients. The results presented herein include data from 35 patients in the previously described Stelten et al. (2018) study, conducted in the Netherlands, along with results from a similar Italian retrospective study [14].

\section{Methods}

Data are reported from two retrospective, single-centre, cohort studies, conducted in the Netherlands ('NL'; CDCA-STUK15-001) and Italy ('IT'; CDCA-STRCH-CR-14-001).

\section{The NL study}

This study was carried out at Canisius-Wilhelmina Hospital in the Netherlands, an official reference centre for CTX patients. Eligible patients were aged 2-75 years, with diagnosed CTX of $\geq 1$-year duration that had been treated with CDCA ( $750 \mathrm{mg} /$ day or $15 \mathrm{mg} / \mathrm{kg} /$ day) orally for $\geq 1$ year. This study used commercially formulated CDCA, which had undergone production via an industrial process, with quality control performed on every batch.

Patients were required to have $\geq 1$ cholestanol and/or urinary bile alcohol assessment $\leq 3$ months prior to treatment with CDCA and $\geq 1$ such value within 2 years from the beginning of therapy with CDCA. Demographic and clinical data were collected prior to CDCA treatment (baseline) and at two post-treatment visits (V1 and V2). To document the patient's current clinical condition (CCC), the most recent available evaluation was also captured during the $\mathrm{CCC}$ visit. The first patient visit was on 27 November 1981 and the last on 3 June 2015. Only those patients who were able to attend hospital visits were included in the analyses presented here.

Baseline characteristics were extracted from medical records. Effectiveness analyses were conducted in the evaluable population, which included all eligible patients with (a) $\geq 1$ quantitative cholestanol assessment the same day or before CDCA initiation, and $\geq 1$ after initiation, or (b) $\geq 1$ qualitative assessment for urinary bile alcohols the same day or before CDCA initiation, and $\geq 1$ after initiation. Effectiveness variables included serum cholestanol, urinary bile alcohols and presence, absence or change in disease signs and symptoms (including psychiatric, cognitive and neurological impairment, epilepsy, polyneuropathy and diarrhoea). The latter were determined through physical examination. Additionally, neurological disability scores (Rankin Scale and EDSS) were reported.

Safety variables were collected in the safety population, which included all eligible patients who had received $\geq 1$ dose of CDCA, regardless of the overall duration of CDCA treatment (i.e. $<1$ or $\geq 1$ year). Safety variables included adverse events (AEs; coded using MedDRA 17.0), serious AEs and laboratory tests.

\section{The IT study}

This study was carried out at the Operative Unit of Neurology and Neurometabolic Disorders, University of Siena, Italy. Eligible patients were aged 2-75 years, with diagnosed CTX of $\geq 1$-year duration that had been treated with CDCA 
( $750 \mathrm{mg} /$ day) orally for $\geq 1$ year. Due to limited availability of commercially produced CDCA prior to licensing, a local pharmacy-produced galenic formulation of CDCA was used in this study.

Patients were required to have $\geq 1$ cholestanol assessment and $\geq 1$ routine laboratory evaluation $\leq 3$ months prior to treatment with CDCA and $\geq 1$ of each within 2 years of treatment initiation. Patients undergoing treatment with other bile acids, statins or steroids $\leq 3$ months before diagnosis, and throughout the follow-up period, were excluded from the study. As in the NL study, demographic and clinical data were collected at baseline, V1, V2 and the CCC visit. The first patient visit was on 3 November 1988 and the last on 22 October 2014.

Baseline characteristics were collected in the screened population by extracting information from medical records. Effectiveness variables were measured in the evaluable population (all patients with $\geq 1$ cholestanol assessment prior to, and $\geq 1$ following, CDCA initiation). Effectiveness variables included serum cholestanol and $7 \alpha$-hydroxy-4-cholesten-3one and presence, absence or change in disease signs and symptoms (including psychiatric, cognitive and neurological impairment and diarrhoea). The latter were determined through physical examination. Additionally, neurological disability scores (Rankin Scale, EDSS) were reported.

Safety variables were measured as per the NL study.

\section{Statistical analyses (both studies)}

Please refer to the Supplemental Material (Online Resource).

\section{Results}

\section{The NL study}

\section{Patient disposition and baseline characteristics}

Thirty-five patients were screened in the study, all of whom formed the safety population and 31 (88.6\%) the evaluable population (Fig. 1). The majority of patients were male $(n=21 ; 60.0 \%)$, with a mean $( \pm$ SD) age of $36.6( \pm 16.8)$ years (Table 1$)$. The median time from baseline visit to V1 was 9.9 months (range: 1.1-186.9), to V2 was 37.6 months (9.5-252.0), and to the CCC visit was 120.9 months (26.2-305.3).

\section{Effectiveness}

Mean serum cholestanol levels significantly decreased from baseline to any post-treatment visit $(p<0.001$ at any visit; Fig. 2a). This was consistent between patients aged $<21$ years or $\geq 21$ years at treatment initiation ( $p<0.001$ at any visit; data not shown).

Neurological impairment was present in $64.5 \%(20 / 31)$ evaluable patients at baseline, $54.8 \%(17 / 31)$ at $\mathrm{V} 1,61.3 \%$ $(19 / 31)$ at V2, and $54.8 \%(17 / 31)$ at the CCC visit. Psychiatric impairment resolved, improved or stabilised in the majority of patients with impairment at baseline $(n=5 / 6$; $85.7 \%$ ). Of 31 evaluable patients, cognitive impairment was present in $58.1 \%(\mathrm{n}=18 / 31)$ at baseline. At V1 and V2, there was improvement or stabilisation in $88.9 \%(n=16 / 18)$ of these
Fig. 1 Patient disposition. Three patients $(10.7 \%)$ in the IT study discontinued, either due to adverse events $(n=1)$ or withdrawal of consent $(n=2)$. Abbreviations: CCC: Current clinical condition; IT: Italy; NL: the Netherlands; V1: Posttreatment visit 1; V2: Posttreatment visit 2
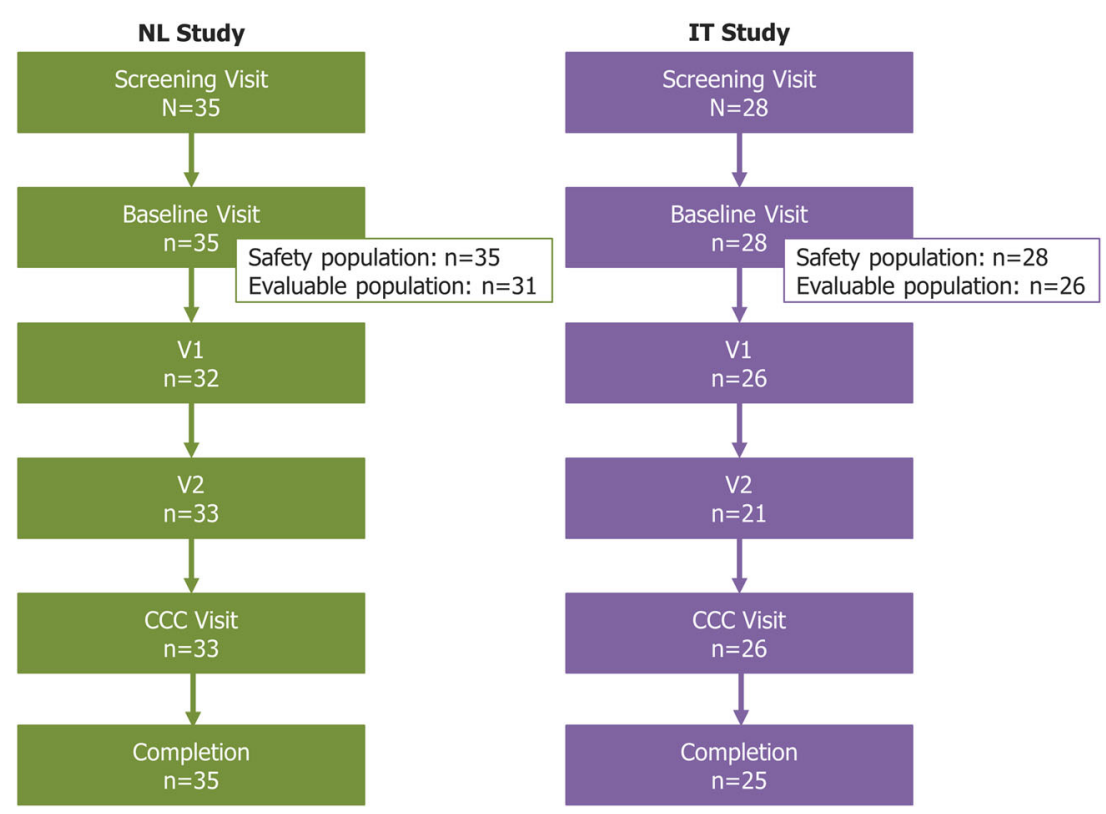
Table 1 Baseline demographics and clinical characteristics

\begin{tabular}{|c|c|c|}
\hline & $\begin{array}{l}\text { NL study } \\
(N=35)\end{array}$ & $\begin{array}{l}\text { IT study } \\
(N=28)\end{array}$ \\
\hline \multicolumn{3}{|l|}{ Demographics } \\
\hline Mean age at disease onset, years $( \pm \mathrm{SD})$ & ${ }^{[\mathrm{a}]}$ & $11.0( \pm 11.2)$ \\
\hline Mean age at diagnosis, years $( \pm$ SD) & $25.6( \pm 13.7)$ & $35.0( \pm 11.4)$ \\
\hline $\begin{array}{l}\text { Mean age at treatment initiation, years }{ }^{[b]} \\
( \pm \text { SD })\end{array}$ & $25.8( \pm 14.0)$ & $35.0( \pm 11.4)$ \\
\hline Mean age at study initiation, years $( \pm \mathrm{SD})$ & $\begin{array}{l}36.6( \pm 16.8) \\
{[n=33]^{\text {cc }}}\end{array}$ & $47.4( \pm 13.2)$ \\
\hline Male, n (\%) & $21.0(60.0)$ & $13.0(46.4)$ \\
\hline \multicolumn{3}{|l|}{ Clinical characteristics $^{[\mathrm{d}]}$} \\
\hline $\begin{array}{l}\text { Mean serum cholestanol levels, } \\
\mu \mathrm{mol} / \mathrm{L}( \pm \mathrm{SD})\end{array}$ & $\begin{array}{l}76.5( \pm 39.0) \\
{[n=27]^{[\mathrm{e}]}}\end{array}$ & $87.8( \pm 39.2)$ \\
\hline $\begin{array}{l}\text { Mean } 7 \alpha \text {-hydroxy-4-cholesten-3-one } \\
\text { level, } \mathrm{mmol} / \mathrm{L}( \pm \mathrm{SD})\end{array}$ & $-^{[\mathrm{f}]}$ & $\begin{array}{l}9.3( \pm 5.7) \\
{[n=16]^{[\mathrm{g}]}}\end{array}$ \\
\hline Neurological impairment present, n (\%) & $20(64.5)$ & $20(76.9)$ \\
\hline Psychiatric impairment present, $\mathrm{n}(\%)$ & $6(19.4)$ & $13(50.0)$ \\
\hline Cognitive impairment present, $\mathrm{n}(\%)$ & $18(58.1)$ & $20(76.9)$ \\
\hline Diarrhoea present, $\mathrm{n}(\%)$ & $23(74.2)$ & $14(53.8)$ \\
\hline Median EDSS score (range) & $\begin{array}{l}1.5(0.0-7.5) \\
{[n=27]^{[\mathrm{h}]}}\end{array}$ & $3.5(0.0-7.0)$ \\
\hline Median Rankin scale score (range) & $\begin{array}{l}1.0(0.0-4.0) \\
{[n=27]^{[\mathrm{h}]}}\end{array}$ & $2.0(0.0-4.0)$ \\
\hline
\end{tabular}

[a] Data not included due to missing data in 32 patients. [b] In the NL study, patients were split into subpopulations based on their age at treatment initiation $(<21$ or $\geq 21$ years). [c] Two patients were deceased when the NL retrospective study was initiated. [d] The total numbers of patients are based on the evaluable population in the NL $(n=31)$ and IT $(n=26)$ studies, unless otherwise stated. [e] Two patients had "elevated" levels and data were missing for two patients. [f] Data not reported. [g] Data were missing for 10 patients. [h] Data were missing for four patients. Abbreviations: EDSS, Expanded Disability Status Scale; IT, Italy; NL, the Netherlands; SD, standard deviation

patients (data missing for 1 patient), and by the CCC visit, cognitive symptoms had resolved, stabilised or improved in all 18 patients with impairment at baseline. Epilepsy resolved, and polyneuropathy stabilised or improved, in all patients with these symptoms at baseline ( $\mathrm{n}=3$ and 11 , respectively). In addition, diarrhoea had resolved by the CCC visit in all patients who reported the symptom at the baseline visit $(n=23 ; 100 \%)$.

Of the 26 patients with Rankin Scale assessments at both baseline and the CCC visit, there was an improvement in $15.4 \%(\mathrm{n}=4)$, stabilisation in $69.2 \%(\mathrm{n}=18)$ and deterioration in $15.4 \%(\mathrm{n}=4)$ patients, although there was no difference in median Rankin Scale score from baseline to the CCC visit ( 0.0 ; range: $-1-$ 2). In addition, the median change from baseline in Rankin Scale score from baseline to the CCC visit was 0.0 (range: $-0.1-0.0$ ) in patients aged $<21$ years at first treatment and 0.0 (range: $0.0-2.0$ ) in patients aged $\geq 21$ years at first treatment.

Of the 26 patients with EDSS scores at baseline and the $\mathrm{CCC}$ visit, there was an improvement in $23.1 \%(\mathrm{n}=6)$, stabilisation in $53.8 \%(n=14)$ and deterioration in $23.1 \%$ $(n=6)$ patients. The median change from baseline in EDSS
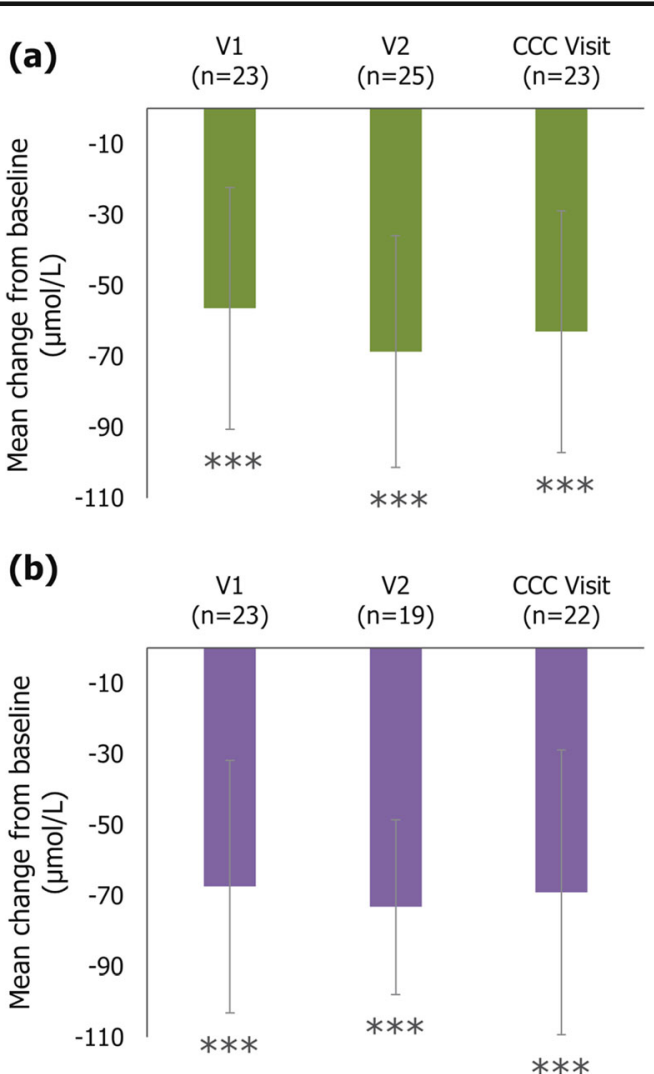

Fig. 2 Mean change from baseline in serum cholestanol level (evaluable populations) (a) NL Study $(N=31)$ (b) IT Study $(N=26)$. Error bars represent the $95 \%$ confidence intervals; $* * * \mathrm{p}<0.001$. The Italian study originally used values in $\mathrm{mg} / \mathrm{dL}$, which have been converted to $\mu \mathrm{mol} / \mathrm{L}$ here. The original mean values $(95 \% \mathrm{CI})$ for each visit in this study were: V1, -2.620 mg/dL (-3.313, -1.928); V2, -2.844 mg/dL (-3.324, -2.365); CCC Visit, $-2.683 \mathrm{mg} / \mathrm{dL}(-3.463,-1.902)$. Abbreviations: CCC: Current clinical condition; IT: Italy; NL: the Netherlands; V1: Post-treatment visit 1; V2: Post-treatment visit 2

score was 0.0 at any post-treatment visit (including when stratified by age at first treatment).

At baseline, all 29 patients with measurements for urinary bile alcohols had elevated levels. These decreased or were normalised in the majority of patients at all post-treatment visits (Supplemental Table 1, Online Resource). No significant difference was observed between the two patient subgroups (data not shown).

\section{Safety}

The median duration of CDCA treatment in the safety population was 9.00 (range: 0.4-26.3) years. A total of 76 AEs were reported in $26(74.3 \%)$ patients (Table 2), including 9 serious AEs in 7 patients $(20.0 \%)$, none of which were fatal or treatment-related. A full listing of AEs and serious AEs can be found in Supplemental Table 2 (Online Resource). Three patients had treatment-related AEs (two cases of constipation and one of toxic hepatitis); none were serious, or of severe intensity. The hepatitis case was an infant and has previously 
Table 2 Summary of adverse events (safety populations)

\begin{tabular}{llllll}
\hline & \multicolumn{2}{l}{ NL study $(N=35)$} & & IT study $(N=28)$ \\
\cline { 2 - 3 } \cline { 5 - 6 } & $n(\%)$ & $\begin{array}{l}\text { Number } \\
\text { of events }\end{array}$ & & $n(\%)$ & $\begin{array}{l}\text { Number } \\
\text { of events }\end{array}$ \\
\hline Any AEs (overall) & $26(74.3)$ & 76 & & $9(32.1)$ & 16 \\
Mild AEs & $23(65.7)$ & 53 & & $1(3.6)$ & 1 \\
Moderate AEs & $15(42.9)$ & 22 & & $5(17.9)$ & 6 \\
Severe AEs & $1(2.9)$ & 1 & & $5(17.9)$ & 9 \\
Discontinuations due to AEs & - & - & & $1(3.6)$ & 1 \\
Treatment-related AEs & $3(8.6)$ & 3 & & $0(0.0)$ & 0 \\
Serious AEs & $7(20.0)$ & 9 & & $9(32.1)$ & 15 \\
\hline
\end{tabular}

Abbreviations: AE, adverse event; IT, Italy; NL, the Netherlands

been reported by Huidekoper et al. (2016) [15]. Treatment was suspended and the event considered treatment-related, although hyperbilirubinaemia was present prior to initiation of treatment. Hepatitis did not recur on re-challenge and ongoing treatment with CDCA at a lower dose of $5 \mathrm{mg} / \mathrm{kg} /$ day.

\section{The IT study}

\section{Patient disposition and baseline characteristics}

Twenty-eight patients were screened and enrolled in the study, all of whom were included in the safety analyses and 26 (92.9\%) in the effectiveness analyses (Fig. 1). The gender split was approximately equal $(46.4 \%$ male $)$, and the mean $( \pm \mathrm{SD})$ age of patients was $47.4( \pm 13.2)$ years (Table 1$)$. The median time from baseline visit to V1 was 23.7 months (range: 5.091.1), to V2 was 52.1 months (17.9-107.1), and to the CCC visit was 76.1 months $(6.4-300.5)$.

\section{Effectiveness}

Mean serum cholestanol levels significantly decreased from baseline to any post-treatment visit $(p<0.001$ at any visit; Fig. $2 b)$. Furthermore, the mean levels of $7 \alpha$-hydroxy-4cholesten-3-one significantly decreased from baseline to V1 and V2 $(p<0.001$; Fig. 3$)$.

Neurological impairment was present in 20 patients (76.9\%) at baseline and had stabilised in $9(45.0 \%)$ and deteriorated in $11(55.0 \%)$ of these patients at the CCC visit. Of the 13 patients who presented with psychiatric impairment at baseline, 92.3\% $(n=12)$ improved or remained stable at the CCC visit. Of 26 evaluable patients, cognitive impairment was present in 20 patients $(76.9 \%)$ at baseline and stabilised in $16(72.7 \%)$ patients at the $\mathrm{CCC}$ visit. In addition, diarrhoea stabilised, improved or disappeared by the $\mathrm{CCC}$ visit in the majority of patients who had this symptom at baseline ( $\mathrm{n}=13 / 14 ; 92.8 \%)$.

There was no deterioration in Rankin Scale score in $61.5 \%(n=16 / 26)$ patients for the entire follow-up period, and

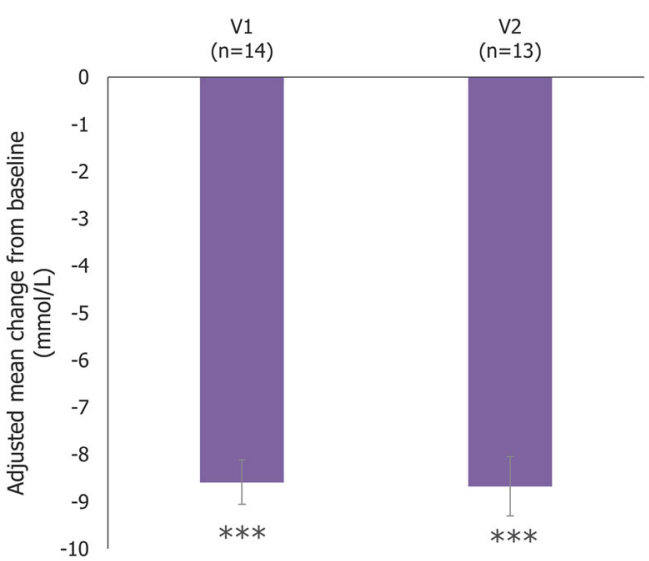

Fig. 3 Mean change from baseline in $7 \alpha$-hydroxy-4-cholesten-3-one level (IT study only; evaluable population). Error bars represent the $95 \%$ confidence intervals; $* * * \mathrm{p}<0.001$. The Italian study originally used values in $\mu \mathrm{g} / \mathrm{dL}$, which have been converted to $\mu \mathrm{mol} / \mathrm{L}$ here. The original mean values $(95 \% \mathrm{CI})$ for each visit in this study were: V1, $343.92 \mu \mathrm{g} / \mathrm{dL}$ (-353.27, -334.58); V2, -347.53 $\mu \mathrm{g} / \mathrm{dL}(-360.08,-334.98)$. Abbreviations: IT: Italy; V1: Post-treatment visit 1; V2: Post-treatment visit 2

there was no difference in the median Rankin Scale score from baseline to the CCC visit ( 0.0 ; range: $0-2)$. Of 26 evaluable patients, EDSS scores remained stable in $46.1 \%(n=12 / 26)$ patients, improved in $3.8 \%(\mathrm{n}=1 / 26)$ patients and deteriorated in $50.0 \%(n=13 / 26)$ patients during the study. The median changes from baseline in EDSS scores were 0.0 at V1 (range: $0.0-2.5$ ) and V2 (range: -0.5-2.0), and 0.5 (range: -0.5-4.0) at the $\mathrm{CCC}$ visit.

\section{Safety}

The median duration of treatment in the safety population was 5.75 (range: 0.0-25.0) years. A total of 16 AEs were reported in 9 patients $(32.1 \%)$, including 15 serious AEs in 9 patients (32.1\%; Table 2). The latter included one fatal case of colon cancer. A full listing of AEs and serious AEs can be found in Supplemental Table 2 (Online Resource). Severe AEs were reported in 5 patients (17.9\%), although no patients had treatment-related AEs.

\section{Discussion}

CTX is a rare neurometabolic disease that results in neurodegeneration, and for which CDCA treatment is the standard of care [4]. The two retrospective studies presented here investigated the safety and effectiveness of CDCA in two cohorts of patients with confirmed CTX and $\geq 1$ year of exposure to the drug. Across both studies, CDCA was generally effective and well-tolerated, with improvement, resolution or stabilisation of the signs and symptoms of disease in most patients. In addition, there 
was some evidence of improvement or stabilisation in disability scale scores, although a number of patients also showed deterioration in these measures. Improvements in the levels of biochemical markers were evident in both cohorts, including serum cholestanol, $7 \alpha$-hydroxy-4cholesten-3-one, and/or urinary bile alcohols. These outcomes reflect those seen in previous studies of CDCA treatment in patients with CTX $[8,14,16-18]$.

Though these observations were broadly comparable between the two studies, there were some differences. For instance, while symptoms such as diarrhoea and neurological, psychiatric and cognitive impairment frequently improved or resolved in the NL study, they generally remained stable in the IT study. Differences in age at diagnosis and treatment initiation may be important reasons for this (patients in the IT study were older and diagnosed later in life on average than those in the NL study), although product differences cannot be ruled out. The observation that mean serum cholestanol levels significantly declined at each visit, in both cohorts, demonstrates the comparable effectiveness of the different formulations and suggests that demographic variation underlies the apparent differences in treatment effectiveness. Inter-study differences were also observed in the Rankin Scale and EDSS scores; this might also be attributable to the older age of patients in the IT study and the greater severity of their symptoms at baseline. Differences between the two studies could also have arisen due to assessment and investigator variabilities, such as in the physical examinations conducted to assess disease signs and symptoms.

In addition to differences in the clinical outcomes, the rate of reported AEs was lower in the IT cohort than the NL cohort. In both studies, AEs were documented and collected on the basis of source documents available at the site, and it is not known whether the differences we report here are real or due to variations in reporting habits. The NL study also included more AEs unrelated to CDCA treatment; however, it remains unclear whether such events were either not reported or not recorded in the IT study.

Beyond the usual limitations associated with retrospective data collection (e.g. missing records and the absence of a control group), a specific limitation of these studies included the use of raw EDSS scores rather than EDSS thresholds, which usually range from 0 to 10 in increments of 0.5 . This means that while improvement in EDSS score was observed for some patients, it is difficult to discern whether these improvements were clinically significant across the evaluable population. Nevertheless, given the chronic and progressive nature of CTX, many patients already have advanced disease at the time of diagnosis, and thus stabilisation of symptoms can be as important as improvement. An additional limitation of these studies was the exclusion of patients in the Netherlands who were institutionalised and therefore unable to attend hospital visits. It is not clear to what extent this might have impacted the observed outcomes.

In summary, the results of these retrospective cohort studies indicate that CDCA is generally effective at reducing cholestanol levels and stabilising or improving the progression of disability and most neurological and nonneurological manifestations. These studies also demonstrate that CDCA has an acceptable safety profile in CTX patients undergoing treatment for a prolonged period of time.

Acknowledgements The authors thank the patients, the investigators and their teams who took part in these studies. The authors also acknowledge Nicole Keijzer and colleagues from CROS NT (Maidenhead, UK) for data monitoring and Patsy Tomlinson, $\mathrm{PhD}$ (Costello Medical, UK), for medical writing and editorial assistance based on the authors' input and direction.

Funding information This study was sponsored by Leadiant Biosciences Ltd (UK). This article was based on the original studies, CDCA-STUK15-001 and CDCA-STRCH-CR-14-001, sponsored by Leadiant Biosciences Ltd (UK). Support for third-party writing assistance for this article, provided by Patsy Tomlinson, $\mathrm{PhD}$ (Costello Medical, UK), was funded by Leadiant Biosciences Ltd (UK), in accordance with Good Publication Practice (GPP3) guidelines (http://www.ismpp.org/gpp3).

\section{Compliance with ethical standards}

Data sharing statement Relevant and anonymised patient-level data and clinical study reports from both studies are available to appropriately qualified individuals upon reasonable request, providing that the proposed use of the data has been independently reviewed and approved. Data will be available immediately following publication, with no end date. Please contact Sue Verma (sue.verma@ leadiantbiosciences.com) to request these data.

Authors' contributions Substantial contributions to study conception and design: AV, MTD, AM, BMLS, SV, AF; substantial contributions to analysis and interpretation of the data: AV, MTD, AM, BMLS, SV, $\mathrm{AF}$; drafting the article or revising it critically for important intellectual content: AV, MTD, AM, BMLS, SV, AF; final approval of the version of the article to be published: AV, MTD, AM, BMLS, SV, AF.

Conflicts of interest $\mathrm{AV}$ and $\mathrm{AM}$ receive honoraria from serving as a consultant for Leadiant Biosciences, Inc. (USA) and Leadiant Biosciences Ltd (UK). MTD and AF received honoraria for attendance at a meeting from Leadiant Biosciences Ltd (UK). BMLS declares to have no conflict of interest. SV is an employee of Leadiant Biosciences Ltd (UK).

Ethics The study protocol, patient information leaflet and informed consent document were approved by the Independent Ethics Committee for both studies. The trials were designed and monitored in accordance with the ethical principles of the International Conference on Harmonisation, the Consolidated Guidelines on Good Clinical Practice, in accordance with the Declaration of Helsinki and following all other requirements of local laws. Written informed consent was obtained from all patients prior to inclusion in the studies.

Open Access This article is licensed under a Creative Commons Attribution 4.0 International License, which permits use, sharing, 
adaptation, distribution and reproduction in any medium or format, as long as you give appropriate credit to the original author(s) and the source, provide a link to the Creative Commons licence, and indicate if changes were made. The images or other third party material in this article are included in the article's Creative Commons licence, unless indicated otherwise in a credit line to the material. If material is not included in the article's Creative Commons licence and your intended use is not permitted by statutory regulation or exceeds the permitted use, you will need to obtain permission directly from the copyright holder. To view a copy of this licence, visit http://creativecommons.org/licenses/by/4.0/.

\section{References}

1. Cali JJ, Hsieh CL, Francke U, Russell DW (1991) Mutations in the bile acid biosynthetic enzyme sterol 27-hydroxylase underlie cerebrotendinous xanthomatosis. J Biol Chem 266:7779-7783

2. Berginer VM, Salen G, P SB (2015) Cerebrotendinous xanthomatosis. In: Rosenberg RN, Pascual JM (eds) Rosenberg's molecular and genetic basis of neurological and psychiatric disease. Elsevier, London

3. Bjorkhem I (2013) Cerebrotendinous xanthomatosis. Curr Opin Lipidol 24:283-287. https://doi.org/10.1097/MOL. 0b013e328362df13

4. Salen G, Steiner RD (2017) Epidemiology, diagnosis, and treatment of cerebrotendinous xanthomatosis (CTX). J Inherit Metab Dis 40: 771-781. https://doi.org/10.1007/s10545-017-0093-8

5. Zádori D, Szpisjak L, Madar L, Varga VE, Csányi B, Bencsik K, Balogh I, Harangi M, Kereszty É, Vécsei L, Klivényi P (2017) Different phenotypes in identical twins with cerebrotendinous xanthomatosis: case series. Neurol Sci 38:481-483. https://doi. org/10.1007/s10072-016-2776-6

6. Mignarri A, Gallus GN, Dotti MT, Federico A (2014) A suspicion index for early diagnosis and treatment of cerebrotendinous xanthomatosis. J Inherit Metab Dis 37:421-429. https://doi.org/ 10.1007/s10545-013-9674-3

7. Lorincz MT, Rainier S, Thomas D, Fink JK (2005) Cerebrotendinous xanthomatosis: possible higher prevalence than previously recognized. Arch Neurol 62:1459-1463. https://doi. org/10.1001/archneur.62.9.1459

8. Duell PB, Salen G, Eichler FS, DeBarber AE, Connor SL, Casaday L, Jayadev S, Kisanuki Y, Lekprasert P, Malloy MJ, Ramdhani RA, Ziajka PE, Quinn JF, Su KG, Geller AS, Diffenderfer MR, Schaefer EJ (2018) Diagnosis, treatment, and clinical outcomes in 43 cases with cerebrotendinous xanthomatosis. J Clin Lipidol 12:11691178. https://doi.org/10.1016/j.jacl.2018.06.008
9. Mignarri A, Magni A, Del Puppo M, Gallus GN, Bjorkhem I, Federico A, Dotti MT (2016) Evaluation of cholesterol metabolism in cerebrotendinous xanthomatosis. J Inherit Metab Dis 39:75-83. https://doi.org/10.1007/s10545-015-9873-1

10. Kuriyama M, Fujiyama J, Kasama T, Osame M (1991) High levels of plant sterols and cholesterol precursors in cerebrotendinous xanthomatosis. J Lipid Res 32:223-229

11. DeBarber AE, Connor WE, Pappu AS, Merkens LS, Steiner RD (2010) ESI-MS/MS quantification of $7 \alpha$-hydroxy-4-cholesten-3one facilitates rapid, convenient diagnostic testing for cerebrotendinous xanthomatosis. Clin Chim Acta 411:43-48. https://doi.org/10.1016/j.cca.2009.09.036

12. Batta AK, Shefer S, Batta M, Salen G (1985) Effect of chenodeoxycholic acid on biliary and urinary bile acids and bile alcohols in cerebrotendinous xanthomatosis; monitoring by high performance liquid chromatography. J Lipid Res 26:690-698

13. Yahalom G, Tsabari R, Molshatzki N, Ephraty L, Cohen H, HassinBaer S (2013) Neurological outcome in cerebrotendinous xanthomatosis treated with chenodeoxycholic acid: early versus late diagnosis. Clin Neuropharmacol 36:78-83. https://doi.org/10.1097/ WNF.0b013e318288076a

14. Stelten BML, Huidekoper HH, van de Warrenburg BPC, Brilstra EH, Hollak CEM, Haak HR, Kluijtmans LAJ, Wevers RA, Verrips A (2019) Long-term treatment effect in cerebrotendinous xanthomatosis depends on age at treatment start. Neurology 92: e83-e95. https://doi.org/10.1212/wnl.0000000000006731

15. Huidekoper HH, Vaz FM, Verrips A, Bosch AM (2016) Hepatotoxicity due to chenodeoxycholic acid supplementation in an infant with cerebrotendinous xanthomatosis: implications for treatment. Eur J Pediatr 175:143-146. https://doi.org/10.1007/ s00431-015-2584-7

16. Amador MM, Masingue M, Debs R, Lamari F, Perlbarg V, Roze E, Degos B, Mochel F (2018) Treatment with chenodeoxycholic acid in cerebrotendinous xanthomatosis: clinical, neurophysiological, and quantitative brain structural outcomes. J Inherit Metab Dis 41: 799-807. https://doi.org/10.1007/s10545-018-0162-7

17. Berginer VM, Salen G, Shefer S (1984) Long-term treatment of cerebrotendinous xanthomatosis with chenodeoxycholic acid. $\mathrm{N}$ Eng1 J Med 311:1649-1652. https://doi.org/10.1056/ nejm198412273112601

18. van Heijst AF, Verrips A, Wevers RA, Cruysberg JR, Renier WO, Tolboom JJ (1998) Treatment and follow-up of children with cerebrotendinous xanthomatosis. Eur J Pediatr 157:313-316. https://doi.org/10.1007/s004310050818

Publisher's note Springer Nature remains neutral with regard to jurisdictional claims in published maps and institutional affiliations. 